\title{
FM 22. Mapping the Frontier Fields with Chandra X-ray Observations
}

\author{
C. Jones, R. van Weeren, G. Ogrean and W. Forman \\ Harvard-Smithsonian Center for Astrophysics \\ 60 Garden St. Cambridge, MA 02138, USA \\ email: cjones@cfa.harvard.edu
}

\begin{abstract}
Chandra has deeply observed the clusters and parallel fields in four Frontier Fields. These observations allow us to dramatically improve our understanding of cluster mergers by comparing the detailed mapping of the hot cluster gas with high resolution mass maps and by identifying merger shocks and cold fronts. In merging clusters, relativistic particles can be re-accelerated to produce radio relics. A comparison of lensing maps and Chandra images allows us to determine the cluster morphology and dynamical state and if there are offsets between the dark matter and the hot gas.
\end{abstract}

Keywords. X-rays: galaxies: clusters, dark matter, acceleration of particles, gravitational lensing, galaxies:peculiar

\section{Introduction}

Deep observations of the Frontier Fields with HST, Chandra, XMM-Newton, Spitzer, and the JVLA allow us to map the distributions of dark matter, cluster galaxies and the hot intracluster medium and to detect, through the high-magnification provided by the massive clusters, high redshift lensed galaxies and AGN. Our primary goals are to 1) understand cluster mergers; 2) compare the distribution of dark matter and baryonic matter, particularly the hot ICM; 3) identify merger shocks by mapping the ICM temperature and pressure; 4) identify and compare radio relics and halos with cluster merger properties to understand particle acceleration in the ICM; 5) determine the number density of very faint radio sources; and 6) understand the effects of mergers on galaxy evolution. This paper presents results for four Frontier Fields clusters MACSJ0717.5+3745, MACSJ0416.1-2403, MACSJ1149.5+2223 and A2744. X-ray images are shown in Fig. 1 (left).

\section{The Frontier Fields Clusters}

MACSJ0717.5+3745 $(z=0.5458)$, the most massive $\left(10^{15} \mathrm{M}_{\text {sun }}\right)$ Frontier Fields cluster, was previously found to be undergoing a quadruple merger event, along with the infall of gas along a filament, and evidence of shock heated gas (Ebeling et al. 2004; Ebeling et al. 2007; Ma et al. 2009, Zitrin et al. 2009, Jauzac et al. 2012). In the radio, MACSJ0717.5+3547 exhibits a $1.5 \mathrm{Mpc}$ halo and an $850 \mathrm{kpc}$ long relic, which combined make this cluster the most luminous extended radio source in the sky (van Weeren et al. 2009, Bonafede et al. 2009). Figure 1 (right) shows that the radio relic lies in a shocked gas region between two massive subclusters, supporting that the relic is produced by the reaccelation of relativistic particles (van Weeren et al. 2015). Due to its large magnified area, deep JVLA observations have yielded more than 50 lensed compact radio sources, 

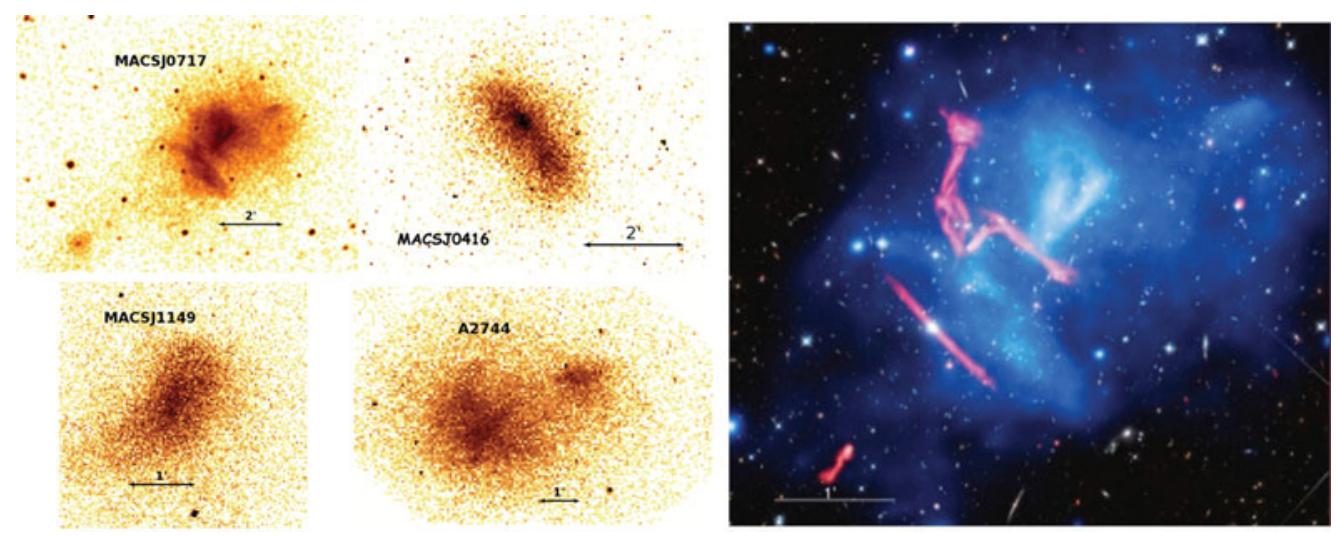

Figure 1. (left) Chandra X-ray images $(0.5-3 \mathrm{keV})$ of the four Frontier Clusters. (right) the X-ray (blue) and radio (red) emission of MACS0717 superposed on the optical image. The radio source southeast of the relic is foreground.

primarily identified with galaxies at $1<z<2$ undergoing star formation at rates of 10 to $50 \mathrm{M}_{\text {sun }}$ per year (van Weeren et al. 2015).

MACSJ0416.1-2403 is a massive merging cluster with an overall ICM temperature of $10 \mathrm{keV}$ and X-ray luminosity of $9 \times 10^{44} \mathrm{ergs} \mathrm{s}^{-1}$ (Mann and Ebeling 2012, Jauzac et al. 2012), Ogrean et al. 2015). From our deeper (365 ks) Chandra observations, Ogrean et al. (2015) found good agreement between the positions of the BCGs in the two primary subclusters and the centroid of each subcluster's X-ray emission. They also determined that the report of an offset between the BCG and the dark matter by Harvey et al. (2015) was caused by a mis-identification of the BCG in the southwestern subcluster.

From deep (365 ks) Chandra observations of MACSJ1149.6+2223, Ogrean et al. (2015) measured a $10.7 \mathrm{keV}$ gas temperature and an X-ray luminosity of $1.6 \times 10^{45} \mathrm{ergs} \mathrm{s}^{-1}$. They also confirmed the radio halo is underluminous, as found by Bonafede et al. (2012).

From Chandra observations of A2744, Owers et al. (2011) determined that the cluster had undergone a major merger which disrupted the cores of both merging subclusters and likely produced "jellyfish" galaxies (Owers et al. 2012).

X-ray and radio studies of the Frontier Fields clusters, along with deep lensing observations, have improved our understanding of the growth of clusters through mergers and have detected lensed populations of high redshift radio and X-ray sources.

\section{References}

Bonafede, A. et al. 2012, MNRAS, 426, 40

Ebeling et al. 2004, ApJ (Letters), 609, L49

Ebeling et al. 2007, ApJ (Letters), 611, L33

Harvey et al. 2015, Science, 347, 1462

Jauzac et al. 2012, MNRAS, 426, 3369

Jauzac et al. 2014, MNRAS, 443, 1549

Mann, C. \& Ebeling, H. 2012, MNRAS, 420, 2120

Ogrean, G. et al. 2015, ApJ, 812, 153

Owers, M., Randall, S., Nulsen, P., Couch, W., David, L., \& Kempner, J. 2011, ApJ, 728, 27

Owers, M., Couch, W., Nulsen, P., \& Randall, S. 2012, ApJ (Letters), 750, L23

van Weeren et al. 2009, A\&\&A, 505, 991

van Weeren et al. 2015, ApJ submitted

Zitrin, A. et al. 2009, ApJ (Letters), 707, L102 